\title{
Imputation of missing network data: Some simple procedures
}

\author{
Mark Huisman* \\ Dept. of Psychology \\ University of Groningen
}

\begin{abstract}
Analysis of social network data is often hampered by non-response and missing data. Recent studies show the negative effects of missing actors and ties on the structural properties of social networks. This means that the results of social network analyses can be severely biased if missing ties were ignored and only complete cases were analyzed. To overcome the problems created by missing data, several treatment methods are proposed in the literature: model-based methods within the framework of exponential random graph models, and imputation methods. In this paper we focus on the latter group of methods, and investigate the use of some simple imputation procedures to handle missing network data. The results of a simulation study show that ignoring the missing data can have large negative effects on structural properties of the network. Missing data treatment based on simple imputation procedures, however, does also have large negative effects and simple imputations can only successfully correct for non-response in a few specific situations.
\end{abstract}

Keywords: Missing data; Single imputation; Descriptive network analysis; Friendship network.

\section{Introduction}

There are several ways in which researchers can cope with missing values, which are frequently found in data collected in empirical research. The easiest option is to simply ignore the missing data and only analyze the observed responses. However, this practice results in (serious) loss of information and a decrease in statistical power, and, more important, may lead to serious bias (e.g., Little and Rubin, 1987; Schafer and Graham, 2002). Other missing data treatments include weighting procedures, model-based procedures (often likelihood-based), and imputation. Much is already known about the effects of missingness on (statistical) data analysis and

\footnotetext{
${ }^{*}$ Department of Psychology, University of Groningen, Grote Kruisstraat 2/1, 9724 TS, Groningen, the Netherlands. Email: j.m.e.huisman@rug.nl, tel: +31 50 3636345, fax: +31 503636304.
} 
the effectiveness of the various treatment procedures (e.g., Little and Rubin, 1987; Schafer and Graham, 2002). However, the effects of missing data on the structural properties of social networks, and especially the treatment of missing network data are scarcely studied.

This paper presents the results of a simulation study that addresses these two issues. First, the effect of missing data on the structure of a network was investigated. The network used in this study is a medium sized friendship network of pupils in a secondary school class. The focus is on missing data caused by non-response of the actors. Second, the performance of some simple imputation techniques to treat the missing network data was inspected by studying the effect of imputation on network level estimates. The paper is organized as follows. In Section 2, the occurrence of missing data in social networks is discussed. Section 3 presents some simple imputation procedures of which the performance is investigated in a simulation study. The design of the simulations is presented in Section 4. Sections 5 and 6 give the results of the study with respect to effects of missingness and effectiveness of the imputation techniques for both undirected and directed networks, respectively. The paper ends with a discussion of the results and an appendix in which two examples are presented. These examples illustrate the performance of the imputation techniques for complex (and more realistic) missing data patterns than were used in the simulation study

\section{Missing data in networks}

Data analysis in social sciences is often hampered by missing data. The analysis of social networks is even more frustrated by missing values, because the complexity of network surveys is more likely to generate missingness, and the analysis and mapping of the structure of the network is especially sensitive to missing data (Burt, 1987a; Ghani, Donnelly, and Garnett, 1998; Borgatti and Molina, 2003; Kossinets, 2006). Social network data consist of a set of actors and a collection of social relations between the actors. In the present paper, the focus is on complete set of actors and a single, binary relation between the actors. For each pair of actors $i, j$, the tie variable $X_{i j}$ indicates whether there is a tie from $i$ to $j\left(X_{i j}=1\right)$ or not $\left(X_{i j}=0\right)$. Self-relations $X_{i i}$ are not defined and set to 0 . The relation can either be directed, from one actor to another, or undirected, in which case $X_{i j}=X_{j i}$. If a network tie or actor is missing, there is limited capacity to describe the network context of the missing actors as well as the context of neighboring actors.

One of the main causes of missingness is non-response ${ }^{1}$. In social network analysis, non-response results in missing network information, that is, missing ties and/or missing scores on actor attributes. Two main types of non-response can be distinguished: unit non-response, where actors are completely missing (i.e., all outgoing ties and attribute scores of an actor), and item non-response, where data on particular items (i.e., particular ties or attributes) are missing. Although missing actors

\footnotetext{
${ }^{1}$ In this paper the terms missing data and non-response will be used interchangeably. See Kossinets (2006) for a detailed discussion of other causes of missingness (e.g., boundary specification problems or fixed choice designs).
} 
seem to be the most relevant to the social network context, missing data on particular ties may occur in whole-network studies when questionnaire instruments incorporate rosters of all actors in a network and a respondent does not indicate the presence or absence of a relation. In longitudinal studies, a third type of non-response can be distinguished: wave non-response (or partial non-response; see Huisman and Steglich, 2008). The present paper is restricted to cross-sectional data, therefore this latter type of non-response is not addressed.

Although non-response results in missing ties for some actors, partial information on the network context of the incompletely observed actors is available: the ties from other actors to the incompletely observed actors are observed. This information can and should be used to assess the effect of the missingness and to adequately analyze the incomplete network (Costenbader and Valente, 2003; Robins, Pattison, and Woolcock, 2004; Gile and Handcock, 2006; Handcock and Gile, 2007). The partial information on the incomplete actors is used to obtain (better) estimates of the structural properties of the actors and the network, and may give information on the nature of the missing data mechanism.

An important question when treating missing data is whether the data are systematically missing, and if so, whether missingness is related to the values of observed variables (properties or attributes). Rubin (1976) defined three types of missing data, related to the level of bias caused by the missingness: Missing Completely at Random (MCAR), Missing at Random (MAR), and Missing Not at Random (MNAR) (see also Schafer and Graham, 2002, or McKnight, McKnight, Sidani, and Figuero, 2007, for a detailed discussion of these definitions).

Data are called Missing Completely at Random if the probability of missingness is not related the observed data, nor to the unknown missing data (that would have been observed). For network data this means that the missingness is unrelated to the value of the missing ties and unrelated to observed data (e.g., observed actor attributes). In this case there is no systematic bias and the observed data are a random subsample from the original set of observations. If the probability of missingness is related to the observed data, but still unrelated to the missing ties, data are called Missing at Random. Although in this case the missing data show systematic patterns, these patterns can be controlled because they are related to observed data in the sample. Given these observed data, the missingness is a random process.

Data are called Missing Not at Random if the probability of missingness is related to the unknown value of the missing ties. This type of missing data has the largest impact on the level of bias in statistical analyses, because it results in systematic differences between respondents and non-respondents. The extent in which structural properties are affected by systematic missing ties partly depends on the properties themselves. Measures based on indegrees, for instance, are found to be more robust against missing data than other measures, because incoming ties are only partially missing (Costenbader and Valente, 2003). 


\section{Imputation}

There are several ways to handle missing data. Two general, popular approaches are likelihood-based estimation based on the available data and imputation (Schafer and Graham, 2002). Butts (2003), Robins, Pattison, and Woolcock (2004), Snijders (2005), Gile and Handcock (2006), Handcock and Gile (2007), and Koskinen (2007) propose methods in the former category. These methods are model-based treatments based on all available data, including the incoming ties of non-respondents, within the framework of statistical network models (e.g., exponential random graph models).

Imputation procedures replace missing values by plausible estimates. This results in a completed data set and gives the researcher the opportunity to proceed with the analysis using standard analysis methods and software. No information is lost, and if the observed data contain information on the missingness, this information can be used to obtain better predictions of the missing values. Schafer and Graham (2002) distinguish four general types of single imputation procedures, that is, imputation procedures in which each missing value is imputed once.

Imputing unconditional means. An easy and simple procedure is replacing each missing item score with the mean over the observed values of that item. In this way the means of the items are preserved, but variances and covariances are often severely biased. Moreover, in case of categorical data, rounding the means may even introduce more bias. In the case of social networks, unconditional means can be computed in several ways: 1) the average number of relations in the network (total mean), that is, the density of the network, 2) the average number of incoming relations of an actor ('item mean'), and 3) the average number of outgoing relations of an actor ('person mean'). For binary network data this results in imputing ones in the case of dense networks, popular actors, and active actors, respectively. Gabbay and Zuckerman (1998) report imputing mean scores and scores reported by other actors for missing ties in a network of work-related interactions between members of $\mathrm{R} \& \mathrm{D}$ laboratories.

Imputing from unconditional distributions. In order to prevent the distribution of the items to be distorted by imputation, procedures are developed that better preserve the data distributions. One class of procedures, known as hot deck procedures, replace each missing value of a respondent, or even the complete non-respondent, with the observed score of a donor respondent from the same data set (e.g., Sande, 1982). Cold deck procedures use donors from other data sets (and studies). These procedures preserve the means and variances of the items in the data set, but still distort the associations between items. In the case of social networks, hot deck imputation means finding a donor actor whose observed ties are used to replace the (completely) missing ties of another actor. Burt (1987b) describes a hot deck imputation procedure for imputation of missing actor attribute data in which donor alters are found "who can speak as a surrogate alter" using the structure of the network (Burt 1987b, p. 1332). Goldstein (1999) uses hot deck imputation for synthesizing kinship networks from household-level survey data.

Imputing conditional means. If there is an association between a missing item and an observed item, the latter may be used to predict the missing value of the for- 
mer item. In non-network data, regression models are often used to predict missing scores. This prediction is the conditional mean of the missing item given the values of the observed item. The procedures gives better estimates of the missing values than unconditional procedures, but underestimates variances and overestimates the relationships between the variables used in the prediction. In social networks, conditional means can be obtained by conditioning on (partially observed) network characteristics like degree, or by using expected values from conditional distributions.

Imputing from conditional distributions. Bias in variances and covariances can be greatly reduced by using a conditional distribution and replacing missing values with draws from this distribution. An often used procedure is imputing regression predictions with an added error term drawn from a normal distribution. Under the assumption that the data are MAR this produces nearly unbiased estimates if the conditional distribution of the missing data given the observed data is correctly specified. For general (arbitrary) patterns of missing data this can be quite complicated. For network analysis there are several classes of distributions available: Ward, Hoff, and Lofdahl (2003) use latent space models (based on logistic regression) to impute missing network data, Steinley and Wasserman (2006) explore the possibilities of conditional uniform random graph distributions (Bernouilli distributions), Handcock and Gile (2007) use exponential random graph models (ERGMs) to produce imputed values.

Apart from the complexity of the latter two classes of imputation methods, single imputation procedures have two major shortcomings. These shortcomings are related to bias and uncertainty. The first problem is that imputations can distort data distributions and relationships, and produce biased estimates, even for data that are MCAR. Second, even if distributions are preserved, single imputation underestimates uncertainty levels, because predictions are treated as observed values and the actual sample size is overestimated. This problem can be solved by multiple imputation (Rubin, 1987). Handcock and Gile (2007) suggest how to obtain multiple imputations using ERGMs.

\subsection{Simple imputation methods}

The simple imputation procedures presented in this section come from the first two classes of procedures defined by Schafer and Graham (2002): imputing unconditional means and imputing from unconditional distributions.

\section{Imputing the unconditional mean}

For binary networks the unconditional total mean (i.e., the average tie value over all observed ties) is equal to the network density. The rounded value of the density (zero if the density is smaller than 0.5) is imputed. This imputation method treats missing ties as absent in sparse networks, and present in dense networks. It is a very crude version of the imputations suggested by Burt (1987a). For ego-centered surveys networks, Burt (1987a) finds that missingness is strongly associated with weak relations, and suggests that they can be replaced by values indicating such weak relations. 


\section{Imputation by reconstruction}

Stork and Richards (1992) suggest reconstructing the missing part of the network using the observed incoming relations of the missing actors. All missing ties $X_{i j}$ are replaced with the observed value of the opposite tie in the dyad: $X_{i j}^{m i s}=X_{j i}$. As the procedure does not allow reconstruction of ties when the dyad is completely missing, additional imputations are necessary. We used random imputation proportional to the observed density (i.e., the probability of a tie is equal to the observed density of the network). This reconstruction procedure was also investigated by Huisman and Steglich (2008) for non-response in network panels.

The reconstruction procedure is the natural candidate for undirected networks as it assumes that reported ties match across actors. For directed networks, however, this assumption is not expected to hold; even in networks with strong reciprocity effects a large number of ties may not be reciprocated. An application of imputation by reconstruction for directed networks with valued ties is given by Gabbay and Zuckerman (1998), who impute the ties reported by observed respondents about their relationships with the non-respondent.

\section{Imputation using preferential attachment}

This procedure was proposed by Huisman and Steglich (2008) and uses the concept of preferential attachment, which states that the probability that an actor will link to another actor is dependent on the connectivity of other actors. (Barabasi and Albert, 1999). Preferential attachment is incorporated in terms of indegrees by assuming that the probability that a missing actor $i$ will be connected to another (observed or missing) actor $j$ is proportional to the indegree of actor $j$ : $\Pi\left(k_{j}\right)=\frac{k_{j}}{\sum_{j} k_{j}}$, with $k_{j}$ the observed indegree of actor $j$, and the sum is over all available actors $j \neq i$. The procedure is expected to preserve the degree distributions. The following steps are taken to replace the missing ties by randomly drawn zeros or ones (see also Huisman and Steglich, 2008):

1. For each actor $i$ with missing ties randomly draw an outdegree $d_{i}$ from the observed outdegree distribution.

2. Determine $J_{i}=\left\{j: j=1, \ldots, n, j \neq i, X_{i j}^{m i s}\right\}$, the set of actors $j$ whose tie from $i, X_{i j}$, is missing. For completely missing actors $i, J_{i}$ contains all other actors in the network.

3. Compute the observed outdegree $d_{i}^{\text {obs }}$ of actor $i$ using the observed ties. For completely missing actors $d_{i}^{\text {obs }}=0$.

4. If $d_{i}>d_{i}^{\text {obs }}$, randomly draw a total of $d_{i}-d_{i}^{\text {obs }}$ actors $j$ without replacement from $J_{i}$ using the the preferential attachment probabilities $\Pi\left(k_{j}\right)$. Impute $X_{i j}=1$ for these actors $j$ and 0 s for the other ties.

\section{Hot Deck imputation}

Using actor attributes and structural properties, completely observed donor actors are found to replace the missing actor (unit non-response) or missing ties of an incomplete actor (item non-response). Actors were matched on a completely observed, 
categorical attribute and on their indegrees by minimizing the absolute differences between the incomplete and donor actors on the two properties (see Section 4 for a description of the network and attribute data). The indegrees were computed from the observed ties of the actors. When no donor actor could be found, zeros were imputed.

\section{Simulation study}

In order to investigate the effect of non-response and imputation on network structure, a simulation study was performed. The general pattern of the study is:

1. 'generate' a complete network,

2. generate missing data by deleting a proportion of actors or ties,

3. analyze the incomplete network data,

4. generate a completed network by imputing the missing ties,

5. re-analyze the completed network data.

Different (independent) factors were used to generate data and missing data: type of network, type of non-response, missing data mechanism, and proportion of missing ties. The incomplete data sets generated were analyzed using descriptive techniques (see Section 4.3) and imputed with the techniques described in Section 3. For each generated data set and imputation method, the procedure is repeated 100 times.

\subsection{Generating network data}

The network data used in the simulation study are a sample data set of 50 actors. These data are provided together with the StOCNET software (Boer, Huisman, Snijders, Steglich, Wichers, and Zeggelink, 2006) and are a subset of the friendship network from the Teenage Health and Lifestyle study, as discussed in Pearson and West (2003) and Steglich, Snijders, and West (2006). The data set consists of girls only, and directed relations between them. In this network, among others, reciprocity effects and network closure effects were found to be strong effects (in network evolution), as well as actor attribute effects related to alcohol consumption. The friendship data were assessed by a name generator allowing for naming up to six friends. Alcohol consumption is coded on a 5-point frequency scale ranging from 1 ('I don't drink') to 5 ('more than once a week'). This data set was also used by Huisman and Steglich (2008) in a simulation study on non-response in longitudinal network studies.

Two types of networks were used in the simulation study: the original, directed network and a symmetrized version of the network. The latter undirected network was created by replacing each tie value with the maximum value of the dyad to which it belongs. 


\subsection{Generating missing data}

For the creation of missing ties two types of non-response were used. The first type is unit non-response, defined as completely missing actors (i.e., all outgoing ties of an actor are missing). The second type is item non-response, defined as occasionally missing ties of observed actors. These two types of missingness, amongst others, were also studied by Kossinets (2006). In Kossinets' study, unit non-response was implemented as completely missing subsets of actors, that is, all outgoing and incoming ties of the actors were deleted. This is different from the definition used in the present paper. In order to compare the results of the present simulations with the results found by Kossinets, a third type of non-response was added: completely missing outgoing and incoming ties of actors. This type was only used to study of the effects of missing data, not to study the imputation methods.

Three missing data mechanisms define the probability that an actor or a tie is missing (see also Huisman and Steglich, 2008):

- Ties are Missing Completely at random (MCAR).

- Missingness is related to an actor attribute: alcohol consumption. The probability of missing is proportional to $\frac{1}{{\text { (alcohol score })^{2}}^{2}}$

- Missingness is related to a network characteristic: outdegree. The probability of missing is proportional to $\frac{1}{(\text { outdegree }+1)^{2}}$.

The three mechanisms are examples of the three definitions by Rubin (1976; see Section 2). The two non-random mechanisms are such that higher scores (high alcohol scores indicate heavier alcohol consumption, high outdegrees indicate more active actors in the network) result in small missingness probabilities ${ }^{2}$. As the attribute alcohol consumption is completely observed for all actors, the data are MAR. The missingness related to outdegree is missing not at random (MNAR), as outdegree is a network characteristic determined from the complete data set (observed and missing).

Missing ties were created according to the two types and three mechanisms. The proportions of missing ties (actors) ranged from 0.10 to 0.90 , in steps of 0.10 .

\subsection{Performance of the imputation techniques}

An imputation technique performs well if it is able to obtain plausible estimates of the missing values and preserves the relationships among the items. This means that analysis of the completed data should result in unbiased estimates of parameters and variances. Moreover, an imputation technique should also reduce the bias caused by the missing data (e.g., see Sande, 1982). The effectiveness of the imputation techniques in reaching these goals is evaluated against some criteria that are frequently used in social network research.

\footnotetext{
${ }^{2}$ This means that the less alcohol respondents consume, the less they are inclined to participate in the study. This may not be overly realistic in every context but does not diminish the usefulness of the mechanism for illustrative purposes.
} 
Huisman and van Duijn (2005) distinguish five classes of procedures that software for social network analysis may or may not possess. Four of these classes contain important criteria (analysis techniques) that can be used to evaluate the effects of missing data and imputation. The groups of procedures are: (1) visualization techniques, (2) descriptive methods to calculate (simple) network statistics, (3) procedure-based analysis based on more complex algorithms, and (4) statistical modeling based on probability distributions. In the present study, the effectiveness of the imputation techniques is investigated using criteria from the second group of procedures: descriptive methods. The focus is on bias in estimating the descriptive statistics, and not on standard errors and inferences.

In order to investigate the impact of missing data and imputation on structural properties of the network the following statistics were used ${ }^{3}$, and were calculated for both undirected and directed networks (e.g., Wasserman and Faust, 1994):

- Mean outdegree.

- Reciprocity. For undirected networks, reciprocity is defined as the proportion of mutual dyads. Because all ties are reciprocated in undirected networks, reciprocity is equal to the density. For directed networks, it is defined as two times the number of mutuals divided by the sum of two times the number of mutuals and the number of asymmetric dyads, $\frac{2 M}{2 M+A}$ (as in the StOCNET software, Boer et al., 2006).

- Transitivity (or clustering; Newman, Strogatz, and Watts, 2001). For undirected networks clustering equals three times the number of triangles divided by the number of connected triples (Newman et al., 2001; this definition is also used in the StOCNET software). For directed networks, transitivity is defined as the ratio of transitive triads to the number of potential transitive triads (StOCNET, Boer et al., 2006).

- Assortativity (Newman, 2003). Assortativity, or assortative mixing on degrees, is defined by Newman (2003) as the correlation between the degrees at either end of an edge, that is, the correlation between degrees of adjacent network nodes. It shows the preference of actors to associate with others who are similar in terms of their volume of connections (Newman, 2003). For directed networks it is the correlation between the indegree of the node the edge leads into and the outdegree of node the edge leads out of.

- Mean inverse geodesic distance. When large amounts of the data are missing in the sparse friendship network, the simulated graphs break up in disconnected components, resulting in undefined (or infinite) geodesics. In order to be able to interpret the results for geodesic distance, the inverse of the geodesic was calculated for both directed and undirected simulated networks, which equals 0 for unconnected nodes.

\footnotetext{
${ }^{3}$ This set of measures does not include important measures for dyadic or nodal properties (e.g., node centrality), which may be affected differently by missingness and imputation, depending on the local neighborhoods of the observed and missing actors. The majority of the statistics were also used by Kossinets (2006), and were chosen to be able to compare the results of the studies
} 
Table 1: Network descriptives for the original friendship networks.

\begin{tabular}{lcc}
\hline & Undirected & Directed \\
\hline Mean degree & 3.240 & 2.320 \\
Reciprocity & 0.066 & 0.603 \\
Clustering/transitivity & 0.209 & 0.373 \\
Assortativity & 0.088 & 0.094 \\
Mean inverse geodesic distance & 0.280 & 0.128 \\
\hline
\end{tabular}

For the original, completely observed network, the values of the descriptives are presented in Table 1. It follows from Table 1 that the two networks do not differ much with respect to the descriptive statistics. The girls' friendship network is rather sparse: the density of the undirected network equals 0.066 (the proportion of mutual dyads; see Table 1), and the density of the directed network equals 0.047 . Also, the mean degrees are small. In the directed network the majority of the ties are reciprocated (reciprocity equals 0.603 ). The networks show a fair amount of transitive relations and a small amount of assortative mixing. On average, the inverse of the geodesic equals 0.280 in the undirected network and 0.128 in the directed network, indicating that it takes a larger number of steps to reach another actor in the directed network. Note that there are two isolated actors.

The relation between alcohol consumption and some aspects of network structure were calculated for a better interpretation of the effect of the missing data mechanisms. The mean alcohol score of the 50 actors is 3.1 ( $\mathrm{SD}=1.2)$, with $38 \%$ of the actors scoring 4 or 5 (indicating high alcohol consumption). The outdegree of the actors with a higher alcohol consumption is somewhat larger than that of less heavy drinkers, 2.7 and 2.1, respectively (the difference is not significant), and the correlation between alcohol consumption and outdegree is 0.16 . No large differences were found in centrality measures between the alcohol groups. Inspection of cliques (of size 3 and larger, containing $74 \%$ of the actors) shows that 8 of the 19 observed cliques almost completely consisted of actors with high alcohol scores (score 4 or 5). The other 8 cliques consist of actors with lower, but similar alcohol scores.

\subsection{Simulation design}

To study the impact of missing data and the performance of the imputation techniques, the two types of networks (directed and undirected) were analyzed separately, using slightly different designs. For directed networks, the design is a complete factorial one, with two types of missing data, three missing data mechanisms, and nine proportions of missing data, resulting in $2 \times 3 \times 9=54$ cells. Within each cell 100 incomplete data sets were generated and repeatedly imputed using the four imputation techniques. The descriptive measures were calculated for the incomplete data and the imputed data sets in each cell.

For undirected networks, an extra type of missing data was added: the complete deletion of subsets of actors. For these actors all outgoing and incoming ties were deleted (cf. Kossinets, 2006). The incomplete data were analyzed using all available 
cases, ignoring the missing ties. This means that, contrary to complete case analysis, actors with some individually missing ties are included in the calculation of the descriptives, and analyses are based on their observed ties only. Moreover, only one imputation technique was used for undirected networks, that is, imputation by reconstruction. The other imputation methods do not take into account the symmetry of the network, and reconstruction is the most logical and easiest method to impute undirected networks. This results in a design with $3 \times 3 \times 9=81$ cells, each in which 100 incomplete data sets were generated and repeatedly imputed using the reconstruction method. The descriptive measures were calculated for the incomplete data and the imputed data sets in each cell.

\section{$5 \quad$ Undirected graphs}

To investigate the effects of missing data and imputation in the networks, the descriptives mean degree, reciprocity, clustering, assortativity and mean inverse geodesic distance were calculated on the simulated incomplete data using the available data, and on the data completed with the imputation methods. The results are presented in Figures 1 and 2, which show the values of the descriptive measures in each cell of the simulation design, and in Table 2, containing the partial eta squared effect sizes from an analysis of variance performed on difference scores: the calculated value of the descriptive measure in a particular cell of the design minus the true value of the original data without missingness.

The figures each contain 10 plots. The plots on the left side show the results for the case of unit non-response (missing actors), the plots on the right side for item non-response (missing ties). The missingness mechanisms are represented by different lines in each plot. The five descriptives are presented from top to bottom on the $y$ axis of the plots, on the $x$ axis corresponds to the proportion of missing actors/ties. Figure 1 depicts how the descriptives change as the proportion missing data increases, where a flat line indicates that missing data do not affect the network structural descriptives.

Table 2 presents the partial eta squared effect sizes for the main effect of method (ignoring the missing data and the imputation methods), type of non-response, missingness mechanism, and proportion of missing data, as well as all two-way interactions. Higher-order interactions were not found to be relevant (except for a few specific cases that are mentioned in the text).

\subsection{Effects of missing data}

Table 2 shows that the proportion of missing data has the largest effect on all five descriptive statistics. There are also relatively large interaction effects including proportion missing, especially the interaction between proportion and (imputation) method. The directions of these effects are shown in Figure 1 and 2, and are discussed below, as well as other effects observed for specific descriptive statistics.

Mean degree. For degree there are large effects of type of non-response, and proportion missing. Both effects are clear in Figure 1. Generally, the bias increases 
Table 2: Partial eta squared effect sizes from the ANOVA of the bias in the descriptive statistics for the symmetrized friendship network. Main effects of Method (Meth), type of non-response (Type), missingness mechanism (Mech), and proportion missing data (Prop), and two-way interaction effects are reported.

\begin{tabular}{lcllll}
\hline & Degree & Recip. & Clust. & Assor. & InvGeo. \\
\hline Meth & 0.734 & 0.072 & 0.010 & 0.026 & 0.955 \\
Type & 0.709 & $0.000^{*}$ & 0.010 & $0.000^{*}$ & 0.171 \\
Mech & 0.091 & 0.024 & 0.010 & 0.008 & 0.106 \\
Prop & 0.850 & 0.202 & 0.317 & 0.259 & 0.957 \\
\hline Meth $\times$ Type & 0.723 & $0.000^{*}$ & 0.005 & $0.000^{*}$ & 0.152 \\
Meth $\times$ Mech & 0.206 & 0.018 & 0.004 & $0.001^{*}$ & 0.524 \\
Meth $\times$ Prop & 0.625 & 0.175 & 0.074 & 0.082 & 0.872 \\
Type $\times$ Mech & 0.242 & $0.000^{*}$ & $0.000^{*}$ & $0.001^{*}$ & $0.001^{*}$ \\
Type $\times$ Prop & 0.640 & $0.000^{*}$ & 0.013 & 0.006 & 0.068 \\
Mech $\times$ Prop & 0.220 & 0.043 & 0.029 & 0.013 & 0.379 \\
\hline * The result was & &
\end{tabular}

* The result was not significant: $p>0.01$.

with more missing data. There is a large decrease in mean degree in case of item nonresponse (i.e., missing ties). In case of unit non-response (i.e., missing actors), degree is not affected or slightly increases, because the parameter controls for network size. In this situation, the non-random mechanisms result in an increase of mean degree, especially degree-related missings, as the actors with lower degrees have a larger probability to be missing and the positive relation between degree and alcohol consumption.

Reciprocity. For reciprocity, the proportion missing data has the largest effect. There is also a small interaction effect between proportion missing and mechanism. Figure 1 shows that reciprocity is stable for randomly missing data, but increases for the degree and alcohol-related mechanisms. The increase is largest for missingness related to degree (as was the case for mean degree), due to the deletion of actors with a smaller number of ties in both non-random mechanisms.

Clustering. Table 2 shows the same effects for clustering as for reciprocity: large effects for proportion missing, and a small interaction effect with mechanism. For random and degree-related missing data, clustering is rather stable up to $60 \%$ missing data, after which the scores generally decrease and the variation in the scores sharply increase. Alcohol-related missingness results in a slight decrease of the clustering. Results for item non-response are similar, only the increase in variation starts at smaller proportions of missing ties, and for large numbers of missing ties the decrease of the clustering coefficient is more pronounced, especially for the MCAR data.

Assortativity. For assortativity the effect of proportion missing is largest, as was the case for the other statistics. Missing data mechanism has a small effect on assortativity (also in interaction with proportion missing), which is different from the effects found for the other statistics. For the alcohol-related missingness, assortativity first increases, and decreases again (after 50\% missing data). This 
indicates that actors with high scores on alcohol consumption have a preference to have ties to others with the same alcohol consumption level (cf. Steglich et al., 2006, who find that alcohol consumption has a strong impact on friendship dynamics in terms of homophily). The other two mechanisms show decreasing scores. These patterns are found for both unit and item non-response.

Inverse geodesic distance. The mean inverse geodesic decreases with higher proportions missing data. This is due to the sparseness of the network, which causes the network to break up into disconnected components. For degree-related non-response the decrease is least steep, as actors with low degrees are missing more often.

The effects of the third type of missingness (i.e., deleting complete subsets of actors) on the mean degree were always between those of unit and item non-response (results are not reported here). Mean degree decreases, but not as sharply as for item nonresponse. The effects on reciprocity and inverse geodesic are the same as for the other two types of non-response, and the effects on clustering largely resemble the effects of the other types. The effects of missingness on assortativity are even better than the effects caused by either unit or item non-response. Assortativity is slightly overestimated, but stable up to $80 \%$ missing, after which it rapidly decreases.

\subsection{Effects of imputation}

The generated incomplete networks were imputed using the reconstruction procedure. The effects of imputation on mean degree, reciprocity, clustering, assortativity, and mean inverse geodesic distance are presented in Figure 2. The figure shows the effects of type of missingness, missing data mechanism, and proportion missing in the same way as Figure 1. Table 2 also presents the effects of missing data treatment on the network descriptives.

Mean degree. The large effect of missing data treatment (as shown in Table 2) is due to the effect of reconstruction in the case of item non-response. The decrease in mean degree due to the missing ties is completely removed by reconstructing the network. The patterns are identical to the patterns for unit non-response (ignoring missingness and reconstructing the missing actors). There is a smaller effect of mechanism, where mean degree increases for the non-random mechanisms and is stable in case of MCAR data.

Reciprocity. For reciprocity similar patterns are found in Figures 1 and 2. Table 2 shows an interaction effect between method and proportion missing. Comparing Figure 1 and 2 shows that the effect of imputation on reciprocity is smaller than the effect of the ignoring the missingness, indicating that reconstruction results in good estimates of reciprocity, even for non-random missingness mechanisms.

Clustering. The effect of reconstruction on clustering is the same for both types of missing data and all three mechanisms. The clustering decreases quickly after $20 \%$ missing data, where the decrease is slower for large proportions missing. When ignoring the missing data (Figure 1) the decrease is smaller indicating that reconstruction results in underestimation of the amount of clustering in the network even for low proportions of missing data. 

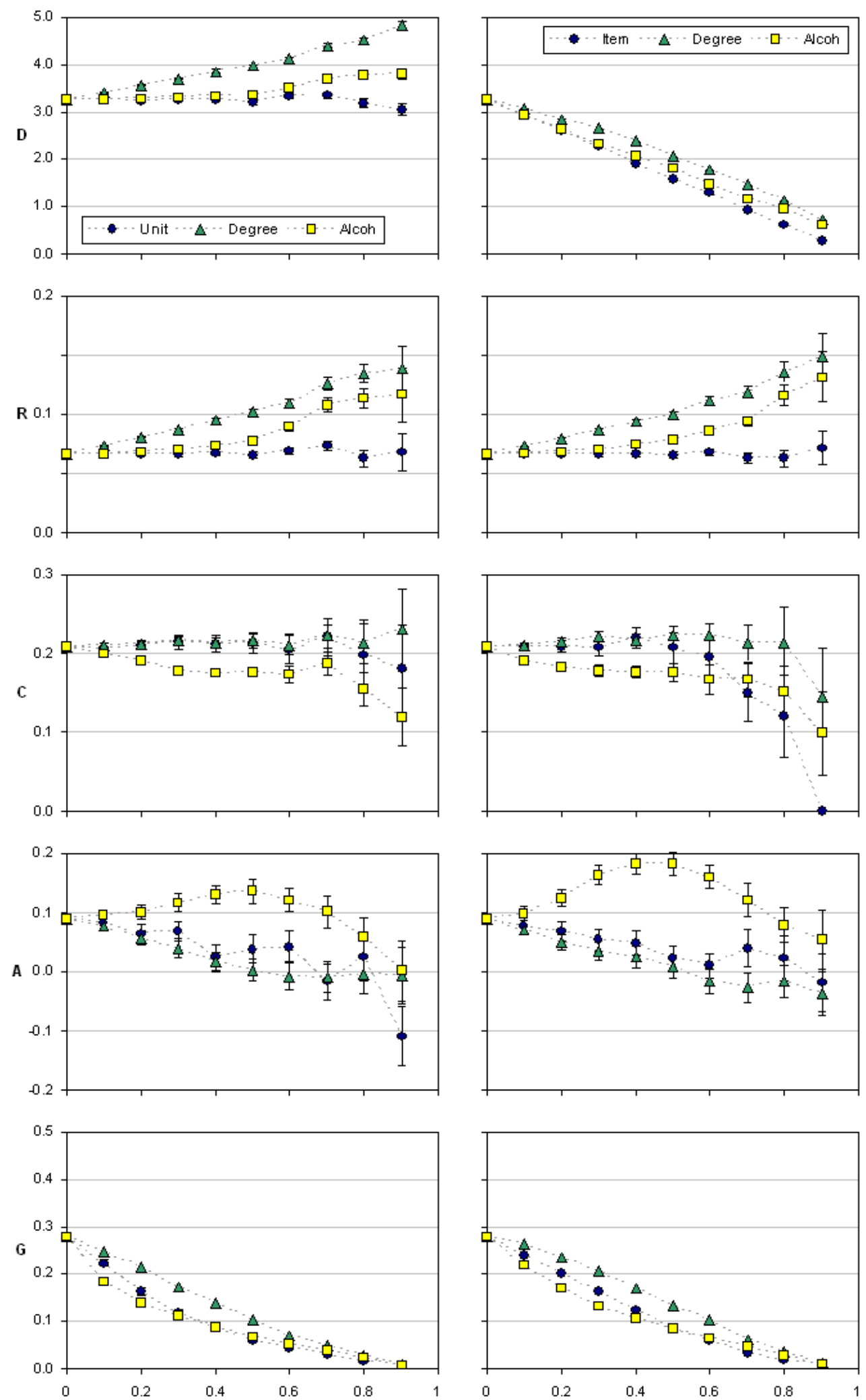

Figure 1: Estimated statistics for the symmetrized, incomplete friendship network (with error bars), for unit non-response (left) and item non-response (right). From top to bottom: plots for degree (D), reciprocity $(\mathrm{R})$, clustering $(\mathrm{C})$, assortativity $(\mathrm{A})$, inverse geodesic $(\mathrm{G})$ on the $y$ axis, and proportion missing data on the $x$ axis. Within each plot three different lines represent the missingness mechanisms: MCAR (labelled Unit or Item), Degree, and Alcohol. 

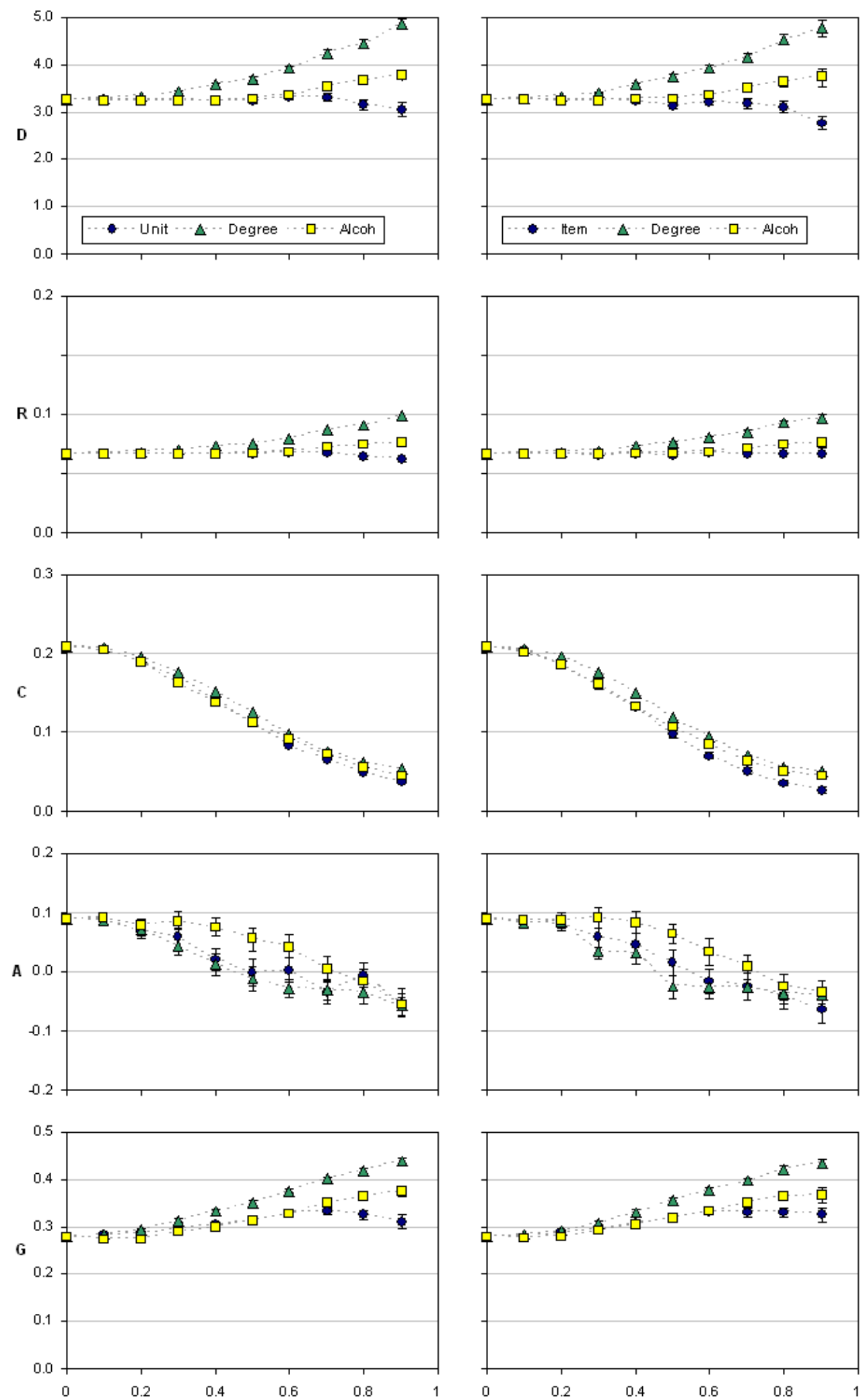

Figure 2: Estimated statistics for the symmetrized friendship network imputed using reconstruction (with error bars), for unit non-response (left) and item non-response (right). From top to bottom: plots for degree (D), reciprocity (R), clustering (C), assortativity (A), inverse geodesic $(\mathrm{G})$ on the $y$ axis, and proportion missing data on the $x$ axis. Within each plot three different lines represent the missingness mechanisms: MCAR (labelled Unit or Item), Degree, and Alcohol. 
Assortativity. Table 2 shows a small effect of method on assortativity, and a larger interaction effect of method and proportion missing. From the figures it follows that these effects are mainly due to alcohol-related missingness: in this case reconstruction results in less bias and stable assortativity estimates for small amounts of missing data (up to $40 \%$ missing).

Inverse geodesic distance. There is a large effect of imputation on the mean inverse geodesic. Where the patterns show decreasing scores when ignoring the missing data, the scores are increasing with proportion missing when imputed. The patterns for unit and item non-response are similar, and the largest bias is found for degree-related missingness. From the figures it follows that reconstruction results in less bias in the inverse geodesic distance than ignoring the missing data.

\section{Directed graphs}

The effects of missing data and imputation on the network properties of directed networks are presented in Figures 3 to 6 and in Table 3. The figures show the values of the descriptive measures in each cell of the simulation design in the same way as for the undirected graphs. Table 3 contains partial eta squared effect sizes from an analysis of variance performed on the difference scores (the calculated value of the descriptive measure in a particular cell of the design minus the true value of the original data without missingness).

\subsection{Effects of missing data}

The effects of missing data on the simulated directed networks largely resemble the effects on undirected networks. The results of the simulations are presented in Figure 3; the main differences with the undirected networks are summarized.

Reciprocity. Reciprocity is stable up to $50 \%$ missing especially for the MCAR data and degree-related missingness. For degree-related missing data the bias is positive, but not as large as in the undirected case, whereas the biases are largest and negative for the alcohol-related mechanism.

Transitivity. The results for transitivity largely resemble those for reciprocity. The degree-related mechanism results in overestimation of transitivity, alcohol-related missing data in underestimation. The decrease in transitivity for undirected networks in situations with high proportions of item non-response, as shown in Figure 1, especially for the MCAR data, is not observed in the directed networks.

Assortativity. In the directed networks the effects of the MCAR mechanism and the degree-related missingness are larger than in the undirected networks. Especially for the degree-related missingness, assortativity decreases faster in directed networks.

\subsection{Effects of imputation}

The generated incomplete networks were imputed using the methods reconstruction, unconditional mean, preferential attachment, and hot deck. The density of the directed network equals 0.05 , which means that imputing the unconditional mean comes down to imputing the value 0 , treating the missing ties as absent. In this 

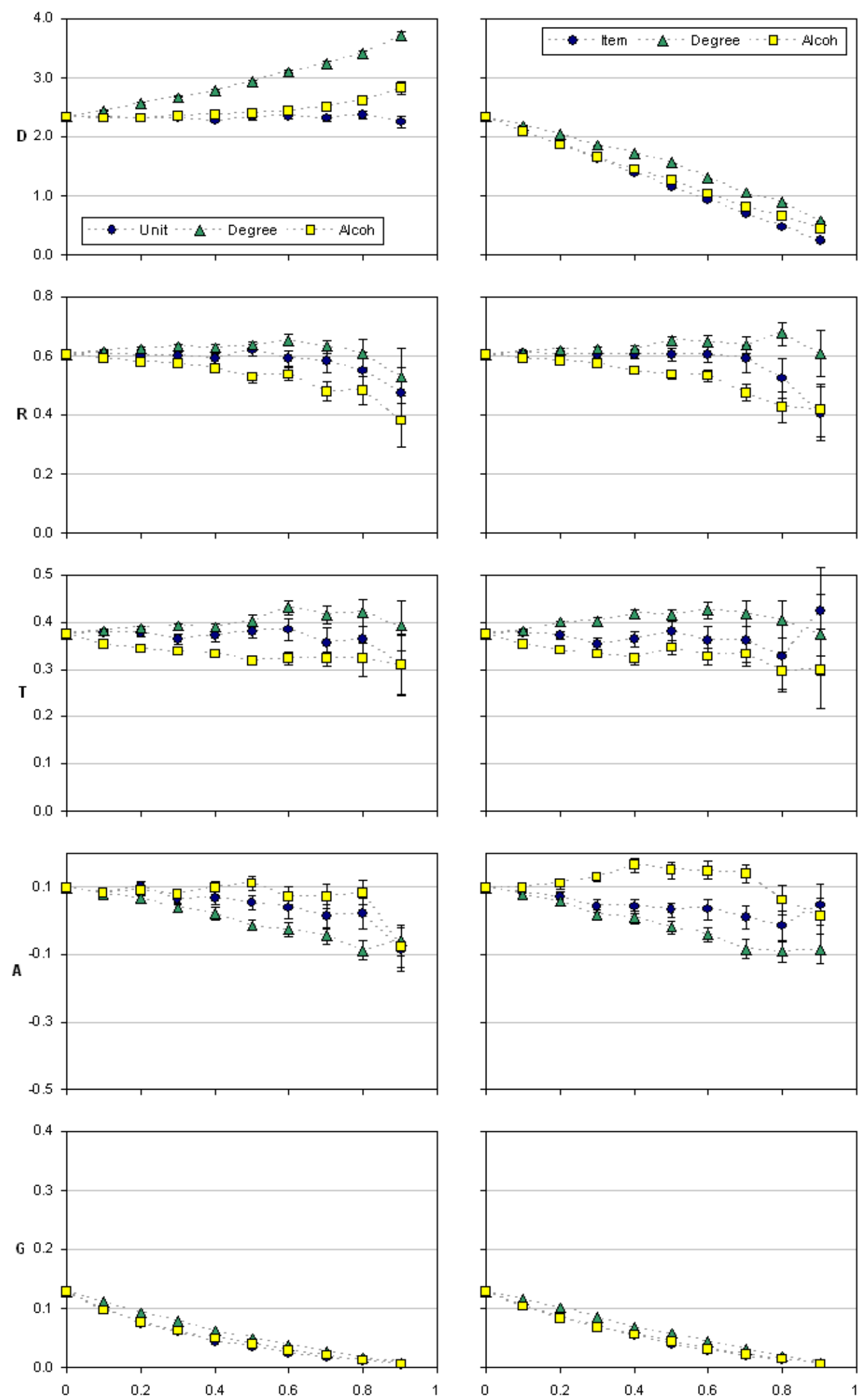

Figure 3: Estimated statistics for the directed, incomplete friendship network (with error bars), for unit non-response (left) and item non-response (right). From top to bottom: plots for degree $(\mathrm{D})$, reciprocity $(\mathrm{R})$, transitivity $(\mathrm{T})$, assortativity $(\mathrm{A})$, geodesic $(\mathrm{G})$ on the $y$ axis, and proportion missing data on the $x$ axis. Within each plot three different lines represent the missingness mechanisms: MCAR (labelled Unit or Item), Degree, and Alcohol. 

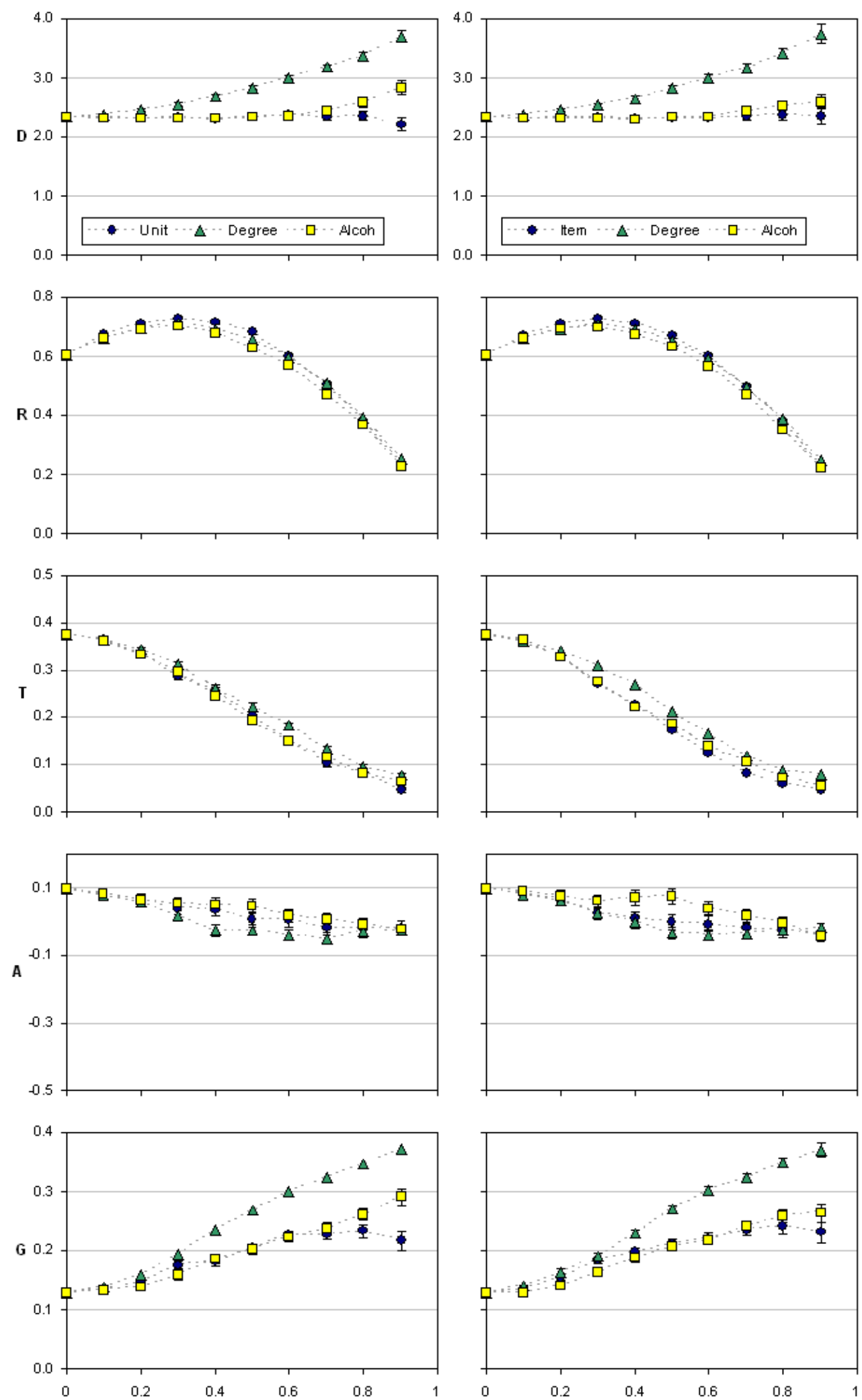

Figure 4: Estimated statistics for the directed friendship network imputed using reconstruction (RE), for unit non-response (left) and item non-response (right). From top to bottom: plots for degree (D), reciprocity $(\mathrm{R})$, transitivity $(\mathrm{T})$, assortativity $(\mathrm{A})$, geodesic $(\mathrm{G})$ on the $y$ axis, and proportion missing data on the $x$ axis. Within each plot three different lines represent the missingness mechanisms: MCAR (labelled Unit or Item), Degree, and Alcohol. 
Table 3: Partial eta squared effect sizes from the ANOVA of the bias in the descriptive statistics for the directed friendship network. Main effects of Method (Meth), type of non-response (Type), missingness mechanism (Mech), and proportion missing data (Prop), and two-way interaction effects are reported.

\begin{tabular}{lccccc}
\hline & Degree & Recip. & Trans. & Assor. & InvGeo. \\
\hline Meth & 0.946 & 0.758 & 0.121 & 0.262 & 0.326 \\
Type & 0.830 & 0.018 & 0.183 & 0.126 & 0.124 \\
Mech & 0.533 & 0.057 & 0.091 & 0.010 & 0.397 \\
Prop & 0.905 & 0.827 & 0.655 & 0.326 & 0.804 \\
\hline Meth $\times$ Type & 0.858 & 0.044 & 0.021 & 0.234 & 0.067 \\
Meth $\times$ Mech & 0.094 & 0.012 & 0.006 & 0.037 & 0.315 \\
Meth $\times$ Prop & 0.880 & 0.535 & 0.205 & 0.135 & 0.481 \\
Type $\times$ Mech & 0.015 & $0.001^{*}$ & 0.014 & 0.025 & $0.002^{*}$ \\
Type $\times$ Prop & 0.779 & 0.026 & 0.107 & 0.058 & 0.183 \\
Mech $\times$ Prop & 0.176 & 0.017 & 0.075 & 0.018 & 0.138 \\
\hline * The
\end{tabular}

* The result was not significant: $p>0.01$.

respect it resembles the available case method, where missing values are ignored. This turns out to be true for assortativity and geodesic distance, where the effects are exactly the same as those for ignoring the missing data. This is also true for mean degree in the situation of item non-response and transitivity for unit non-response. In the other two cases (mean degree for unit non-response and transitivity for item non-response) the effects are negative: there is a sharp and almost linear decrease. The results are not reported here.

The results of the simulations are summarized in Table 3, using partial eta's squared. The effects of the procedures reconstruction (RE), preferential attachment (PA), and hot deck (HD) on the network properties are presented in Figures 4, 5, and 6 , respectively. It should be noted that in case of item non-response the hot deck method frequently was not able to find donor actors to replace the missing ties. For missingness levels of 0.5 and higher suitable donors were not available in the network and zeros were imputed. For these situations the hot deck method and the unconditional mean method show the same effects.

Mean outdegree. Table 3 shows large effects of all factors on mean outdegree. There is a large three-way effect of imputation method, type of non-response, and proportion missing (not reported in Table 3), but clearly visible in the figures. For unit non-response the patterns resemble those of ignoring the missing data, indicating that imputation does not lead to better results in this situation. In case of item non-response, the patterns for RE are clearly different from the other methods. Here, the downward effect of missingness (Figure 3) is corrected. The methods PA and HD somewhat correct for small proportions missing (HD even over-corrects), after which they show the same effect as ignoring the missingness. In all plots, the largest positive bias is found for degree-related missing data

Reciprocity. For reciprocity there is a large interaction effect of method and proportion missing. Ignoring the missing data gives reasonably stable results (for 

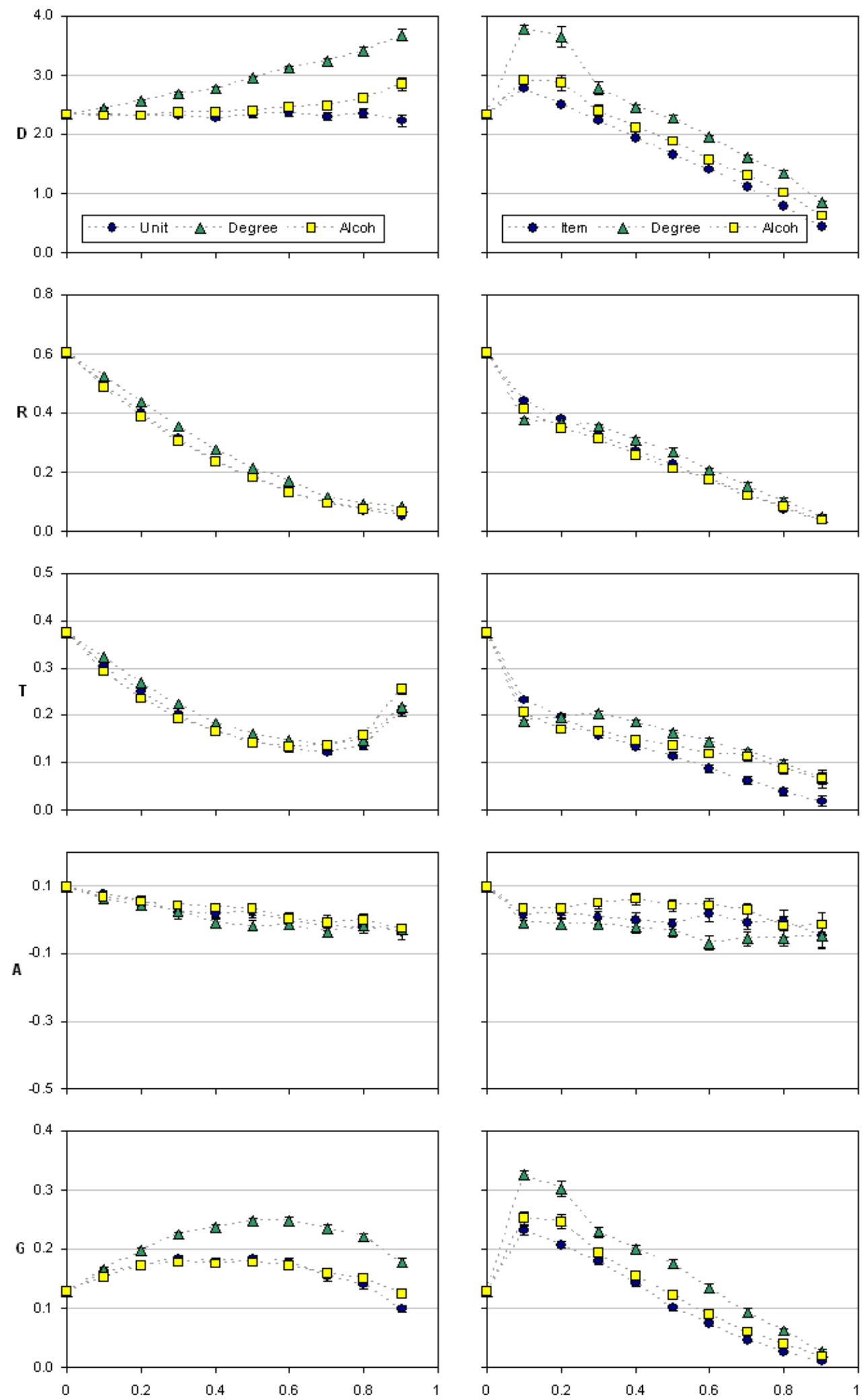

Figure 5: Estimated statistics for the directed friendship network imputed using preferential attachment (PA), for unit non-response (left) and item non-response (right). From top to bottom: plots for degree $(\mathrm{D})$, reciprocity $(\mathrm{R})$, transitivity $(\mathrm{T})$, assortativity $(\mathrm{A})$, geodesic $(\mathrm{G})$ on the $y$ axis, and proportion missing data on the $x$ axis. Within each plot three different lines represent the missingness mechanisms: MCAR (labelled Unit or Item), Degree, and Alcohol. 

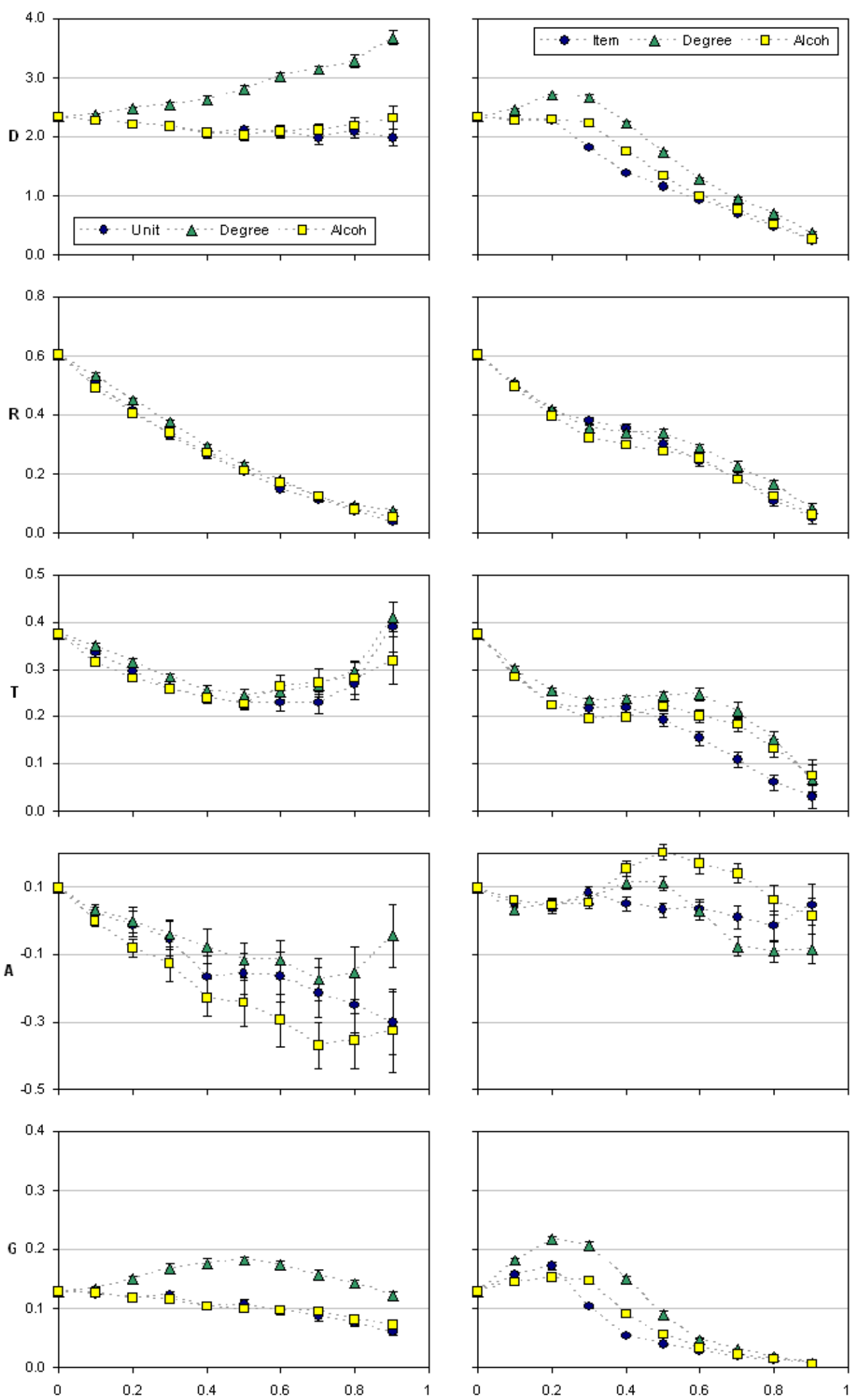

Figure 6: Estimated statistics for the directed friendship network imputed using hot deck (HD), for unit non-response (left) and item non-response (right). From top to bottom: plots for degree $(\mathrm{D})$, reciprocity $(\mathrm{R})$, transitivity $(\mathrm{T})$, assortativity $(\mathrm{A})$, geodesic $(\mathrm{G})$ on the $y$ axis, and proportion missing data on the $x$ axis. Within each plot three different lines represent the missingness mechanisms: MCAR (labelled Unit or Item), Degree, and Alcohol. 
small to medium proportions missing), but the imputation methods show different patterns. The PA and HD methods show similar effects: reciprocity is increasingly underestimated for higher proportions of missing data. RE shows a different (quadratic) pattern: reciprocity is overestimated for small proportions missing, underestimated for larger proportions. This latter result is largely due to the random character of the method when many ties are missing. The figures show that there are no differences between the two types of non-response and the three missingness mechanisms.

Transitivity. The results for transitivity largely resemble those for reciprocity. Table 3 shows a large interaction effect of method and proportion missing. The imputation methods result in underestimated transitivity scores, whereas ignoring the missing data gives more or less stable results. Generally, the negative bias increases with proportion missing data. Only hot deck imputation for unit nonresponse shows an increase in scores (after an initial decrease). This is probably due to the imputation of zeros for large proportions missing (as no suitable donors can be found), which leads to the same results as found in Figure 3 for ignoring the missing data.

Assortativity. Table 3 presents two interaction effects for assortativity: the largest of method and type, and a second of method and proportion missing. The general picture is clear from Figures 4 to 6: assortativity gradually decreases for higher proportions missing. The methods RE and PA give similar, reasonably stable results, especially for small amounts of missing data. Here, the bias is generally largest for degree-related missingness and smallest for alcohol-related missingness. The HD method, however, shows a large decrease in the assortativity score in case of missing actors, and more erratic behavior in case of missing ties. Moreover, the error bars show large variations in the scores, making HD not a very good method when estimating assortativity.

Inverse geodesic distance. For the mean inverse geodesic distance there is a large tree-way interaction effect for method, mechanism, and proportion missing (not reported in Table 3). From the figures it follows that degree-related missing data results in large positive bias, especially for the RE and PA methods. For RE the bias increases with higher proportions, whereas for the other methods the bias starts decrease for high proportions missing data. The RE method overcorrects the decrease in assortativity found for ignoring the missing data (Figure 3). The other two methods overcorrect for small amounts of missing data, but tend to undercorrect for large numbers of missing ties.

In the appendix two examples are presented of the directed network data with more complex missing data mechanisms than were used in the simulation study.

\section{Discussion}

In this paper, a simulation study was performed to investigate the effect of nonresponse on the structural properties of social networks, and the ability of some simple imputation techniques to treat the missing network data. The simulations 
were based on an existing friendship network in school classes, and missing data were created using different types, mechanisms, and proportions of missing data. In simulation studies like this, the question always is how realistic the design and conditions of the study are, in order to suggest real-life applicability of the results and generalization to situations that were not explored.

The data set used in the simulations was chosen to be the empirical data set that was used by Huisman and Steglich (2008), consisting of a medium-sized, sparse network. This makes generalizing the results of the simulations to denser networks difficult, and such networks need further study. Although item non-response (missing ties) is probably less common in network surveys than unit non-response (missing actors), both types of missingness were studied. The missing data mechanisms included a random and two non-random mechanisms, which result in missingness related to actor and network characteristics. Although the occurrence of these latter mechanisms in real data was not inspected, they seem plausible and serve to avoid big simplifications by modelling all mechanisms as random.

The selected outcome measures are a sample of descriptive measures that summarize structural properties of the network and do not include measures of nodal properties or specific ties. The effect of missing data and imputation on these latter properties depend on the local neighborhoods of the specific ties, which are not studied in this paper.

The imputation methods that were studied are simple methods which either follow logically from the general classification given by Schafer and Graham (2002), or are proposed and/or used by others (Burt, 1987a, 1987b; Stork and Richards, 1992; Gabbay and Zuckerman, 1998; Goldstein, 1999; Huisman and Steglich, 2008). Some of the methods needed slight (ad hoc) modifications in order to work in all situations. These are the random imputation of values in the reconstruction method when both ties in the dyad are missing, and imputing zeros in the hot deck method when no donor actor is available. These situations occur when the proportions missing data are (unrealistically) high and are therefore expected not to distort the results too much.

\section{Effects of non-response}

The simulation study shows that ignoring missing data in networks can have large effects on descriptive analyses of social networks depending on the type of network and type of non-response. Comparing directed and undirected graphs (Figures 1 and 3 ), the direction of effects of non-response on the descriptive statistics are generally the same. For undirected networks biases are larger for the degree-related statistics (mean degree, inverse geodesic), for directed networks the biases are somewhat larger for reciprocity and clustering.

Comparing unit non-response (missing actors) and item non-response (missing ties), the patterns are also generally the same, with item non-response resulting in slightly more bias. One exception is mean degree, which is more or less stable for unit non-response, whereas item non-response results in lower degrees. The missing data mechanisms have a somewhat smaller effect, where generally (one of) the non-random mechanisms results in the largest biases. Especially the degreerelated missingness leads to large biases in estimating degree-related statistics. 
The results found by Kossinets (2006) were similar for degree and assortativity in those situations where the definitions of the mechanisms agree. The effect on clustering and geodesic distance is different. The differences are mainly due to differences in the networks used in the studies. In Kossinets' study the networks are (very) large and have larger values for mean degree, clustering, assortativity, and mean path length. Moreover, the networks used by Kossinets are one-mode projections of bipartite graphs.

\section{Effects of imputation}

For low to medium proportions missing data in undirected networks, imputation by reconstruction is quite capable of correcting the effects of non-response. Reconstruction results in stable estimates of reciprocity (as expected), mean degrees (especially in case of item non-response), and inverse geodesic, up to $40 \%$ missing data. Also the results for assortativity are at least as good as ignoring the missing data. Only for clustering the bias increases rapidly for higher proportions missing.

In directed networks, imputation by reconstruction does not correct the effects of non-response as well as in undirected networks. Here the biases are usually larger than in the undirected networks, especially for the estimates of reciprocity. Generally, ignoring the missing data gives smaller biases, except for mean degree in case of item non-response.

For directed networks, generally reconstruction is the best of the studied imputation methods. This is confirmed by the two examples in the appendix. The other two procedures, preferential attachment and hot deck, generally result in more bias than reconstruction. Imputation using preferential attachment was expected to preserve the degree distribution of the network. However, it cannot adequately correct for biases, and does not give better results than reconstruction (except in a few cases with high proportions missing, which are quite unrealistic). This results was also found by Huisman and Steglich (2008).

The performance of the hot deck procedure depends on the availability of donor actors and on the relation between the descriptive statistic (structural network property) and the matching variable. For medium and high proportions missing, hot deck breaks down because no suitable donors can be found, especially in case of item nonresponse. For low proportions missing hot deck results in small biases for inverse geodesic and to a lesser extent mean degree.

The simulations show that for low proportions of missing data, generally the bias in the descriptive statistics is not very large. This suggests that simply ignoring the missing data does not lead to seriously biased results in the studied descriptive statistics. Moreover, the simple imputation procedures generally are not able to correct these biases, especially for medium to large amounts of missing data. This was also shown in the examples in which more complex missingness mechanisms were presented.

Does this mean that the remark of Robins et al. (2004) that imputation is unlikely to be very successful ${ }^{4}$, is true? Even for the simple methods used in this study, the

\footnotetext{
${ }^{4}$ The remark was made with respect to unit non-response, but the question can and should be
} 
answer should be no, because the reconstruction method proposed by Stork and Richards (1992) can correct the effects of non-response in some situations.

In the simulations, reconstruction is the best simple imputation method. For undirected networks and small amounts of missing data (20-30\%), it gives better results than ignoring the missing data, regardless the missing data mechanism. And even for larger amounts of missing data (50\%), reconstruction is the recommended procedure when calculating mean degree, reciprocity, and inverse geodesic.

In directed networks, the measures most affected by non-response are degree and inverse geodesic. For these measures reconstruction gives good results when the proportion of missing data is low. For the other measures ignoring the missing data is better than imputation (using simple methods), and even gives good results for small amounts of missingness: reciprocity, transitivity, and assortativity are fairly stable up to $40 \%$ missing data.

It should be noted that for large amounts of missing data, the reconstruction method often fails, because both ties in a dyad are missing. In this situation, the ties are randomly imputed, conditional on the observed density. This affects the structure of the network as nodes are usually not randomly connected and it influences clustering in the neighborhood of the new tie. Because the existing neighborhood structure is not well preserved, imputation methods that work at a global level are likely to be weaker than intended.

This also holds for the other simple, ad hoc imputation methods studied in the simulations, and generally they do not give good results. They distort data distributions and structural properties, especially for larger amounts of missing data (cf. Schafer and Graham, 2002). The size and sparseness of the network may also have lessened the effectiveness of the imputations, because changing a small number of ties can have a large impact on the overall structure of the network.

\section{References}

Barabasi, A-L. and Albert, R. (1999). Emergence of scaling in random networks. Science, 286, 509-512.

Boer, P., Huisman, M., Snijders, T.A.B., Steglich, C.E.G., Wichers, L.H.Y., and Zeggelink, E.P.H. (2006). StOCNET: An open software system for the advanced statistical analysis of social networks. Version 1.7. Groningen: ICS / SiencePlus. http://stat.gamma.rug.nl/stocnet/.

Borgatti, S.P. and Molina, J.L. (2003). Ethical and strategic issues in organizational social network analysis. Journal of Applied Behavioral Science, 39, 337-349.

Burt, R.S. (1987a). A note on missing network data in the general social survey. Social Networks, 9, 63-73.

Burt, R.S. (1987b). Social contagion and innovation: Cohesion versus structural equivalence. The American Journal of Sociology, 92, 1287-1335.

Butts, C.T. (2003). Network inference, error, and informant (in)accuracy: a Bayesian approach. Social Networks, 25, 103-140.

Costenbader, E. and Valente, T.W. (2003). The stability of centrality measures when networks are sampled. Social Networks, 25, 283-307.

generalized to all types of missing data. 
Gabbay, S.M. and Zuckerman, E.W. (1998). Social capital and opportunity in corporate R\&D: The contingent effect of contact density on mobility expectations. Social Science Research, 27, 189-217.

Ghani, A.C., Donnelly, C.A. and Garnett, G.P. (1998). Sampling biases and missing data in explorations of sexual partner networks for the spread of sexually transmitted diseases. Statistics in Medicine, 17, 2079-2097.

Gile, K. and Handcock, M.S. (2006). Model-based assessment of the impact of missing data on inference for networks. CSSS Working paper no. 66, University of Washington, Seattle. (http://www.csss.washington.edu/Papers/wp66.pdf)

Goldstein, J.R. (1999). Kinship networks that cross racial lines: The exception or the rule? Demography, 36, 399-407.

Handcock, M.S. and Gile, K. (2007). Modeling social networks with sampled or missing data. CSSS Working paper no. 75, University of Washington, Seattle. (http://www.csss.washington.edu/Papers/wp75.pdf)

Huisman, M. and Steglich, C.E.G. (2008). Treatment of non-response in longitudinal network studies. Social Networks, 30, 297-308.

Huisman, M. and van Duijn, M.A.J. (2005). Software for social network analysis. In Carrington, P.J., Scott, J., and Wasserman, S. (Eds.), Models and Methods in Social Network Analysis, pp. 270-316. Cambridge University Press, Cambridge.

Kossinets, G. (2006). Effects of missing data in social networks. Social Networks, 28, 247268.

Koskinen, J. (2007). Fitting models to social networks with missing data. Paper presented at Sunbelt XXVII, the International Sunbelt Social Network Conference, May 1-6, 2007, Corfu, Greece.

Little, R.A.J. and Rubin, D.B. (1987). Statistical Analysis with Missing Data. New York: Wiley.

McKnight, P.E., McKnight, K.M., Sidani, S., and Figueredo, A.J. (2007). Missing Data. A Gentle Introduction. New York: Guildford Press.

Newman, M.E.J. (2003). Mixing patterns in networks. Physical Review E, 67, 026126.

Newman, M.E.J., Strogatz, S.H., and Watts, D.J. (2001). Random graphs with arbitrary degree distributions and their applications. Physical Review E, 64, 026118.

Pearson, M. and West, P. (2003). Drifting smoke rings: social network analysis and Markov processes in a longitudinal study of friendship groups and risk-taking. Connections, 25, 59-76.

Robins, G., Pattison, P., and Woolcock, J. (2004). Missing data in networks: exponential random graph $(p *)$ models for networks with non-respondents. Social Networks, 26, $257-283$.

Rubin, D.B. (1976). Inference and missing data. Biometrika, 63, 581-592.

Rubin, D.B. (1987). Multiple Imputation for Nonresponse in Surveys. New York: Wiley.

Sande, I.G. (1982). Imputation in surveys: Coping with reality. The American Statistician, $36,145-152$.

Schafer, J.L. and Graham, J.W. (2002). Missing data: our view of the state of the art. Psychological Methods, 7, 147-177.

Snijders, T.A.B. (2005). Models for longitudinal network data. In Carrington, P.J., Scott, J., and Wasserman, S. (Eds.), Models and Methods in Social Network Analysis, pp. 215-247. Cambridge University Press, Cambridge. 
Steglich, C.E.G., Snijders, T.A.B., and West, P. (2006). Applying SIENA: An illustrative analysis of the co-evolution of adolescents' friendship networks, taste in music, and alcohol consumption. Methodology, 2, 48-56.

Steinley, D. and Wasserman, S. (2006). Approximate distributions of several common graph statistics: hypothesis testing applied to a terrorist network. Proceedings of the American Statistical Association, Statistical Applications in Defense and National Security. Santa Monica, CA: Rand Corporation.

Stork, D. and Richards, W.D. (1992). Nonrespondents in communication network studies. Group \& Organization Management, 17, 193-209.

Van de Bunt, G.G. (1999). Friends by choice. An actor-oriented statistical network model for friendship networks through time. Amsterdam: Thesis Publishers.

Ward, M.D., Hoff, P.D., and Lofdahl, C.L. (2003). Identifying international networks: Latent spaces and imputation. In Breiger, R., Carley, k., and Pattison, P. (Eds.), $D y$ namic Social Network Modelling and Analysis: Workshop Summary and Papers, pp. 345-360. Washington: The National Academic Press.

Wasserman, S. and Faust, K. (1994). Social Network Analysis. Methods and applications. Cambridge: Cambridge University Press. 


\section{APPENDIX: Two examples}

In this appendix two examples are presented to illustrate the effects of missing data and the imputation methods presented in Section 3 when applied to more complex missingness mechanisms. First, a mechanism is simulated that is a mix of the mechanisms used in the simulation study. Next, an empirical mechanism is studied by applying the imputation methods to empirical incomplete network data.

\section{First example: Simulated mechanism}

The first example uses the directed girls' friendship data that were also used in the simulations. Missing data were generated by combining different types of nonresponse and missingness mechanisms in the following (arbitrary) way: first delete $5 \%$ of the actors at random (MCAR), next, delete about $25 \%$ of the actors proportional to outdegree, and finally, delete about $5 \%$ of the ties proportional to alcohol consumption.

This process was repeated 100 times, resulting in an average fraction of missing ties of 0.36 in the generated data sets. The proportion of missing data in the data set is fairly high (although such numbers may be encountered in real data) in order to clearly illustrate the impact of missing data and imputation. Each of the incomplete data sets was imputed with the four imputation procedures described in Section 3. The effects of missingness and imputation were inspected by calculating the mean degree, reciprocity transitivity, assortativity, and mean geodesic distance. The results are presented in Figure 7.

The boxplots in Figure 7 are consistent with the results of the simulation study in Figures 3 to 6 . Degree-related measures are generally overestimated (mean degree, inverse geodesic), as is reciprocity in case of imputation by reconstruction, and the other statistics are underestimated. The strong dependence of missingness on degree is reflected by almost all methods, especially the methods that preserve the degree distribution (cf. the results of the simulation study). Ignoring the missing data (using available cases only) gives good results, and gives in almost all situations better results than the imputation procedures. The effects on reciprocity and transitivity are large, especially for preferential attachment. This latter procedure, together with hot deck, behaves poorly for all descriptives.

\section{Second example: Empirical data set}

The second example uses another friendship network that is distributed together with the StOCNET software. It is the sixth observation of a network of 32 freshmen students at a university in the Netherlands (van de Bunt, 1999). The studied relation is defined as a friendly relation. Three actor attributes are available, of which one is used: the length of the programme followed by the students (2, 3, 4 years).

Of the 32 actors in this observation of the network, 7 are completely missing (unit non-response), and 3 actors have one additional missing tie (item non-response). This results in 220 missing ties, which is $22.2 \%$ of the ties. This amount of missing data is not uncommon in empirical research, especially in longitudinal studies. The missing ties were imputed using the four imputation procedures, and the effects of imputation on the descriptive statistics are presented in Table 4. 

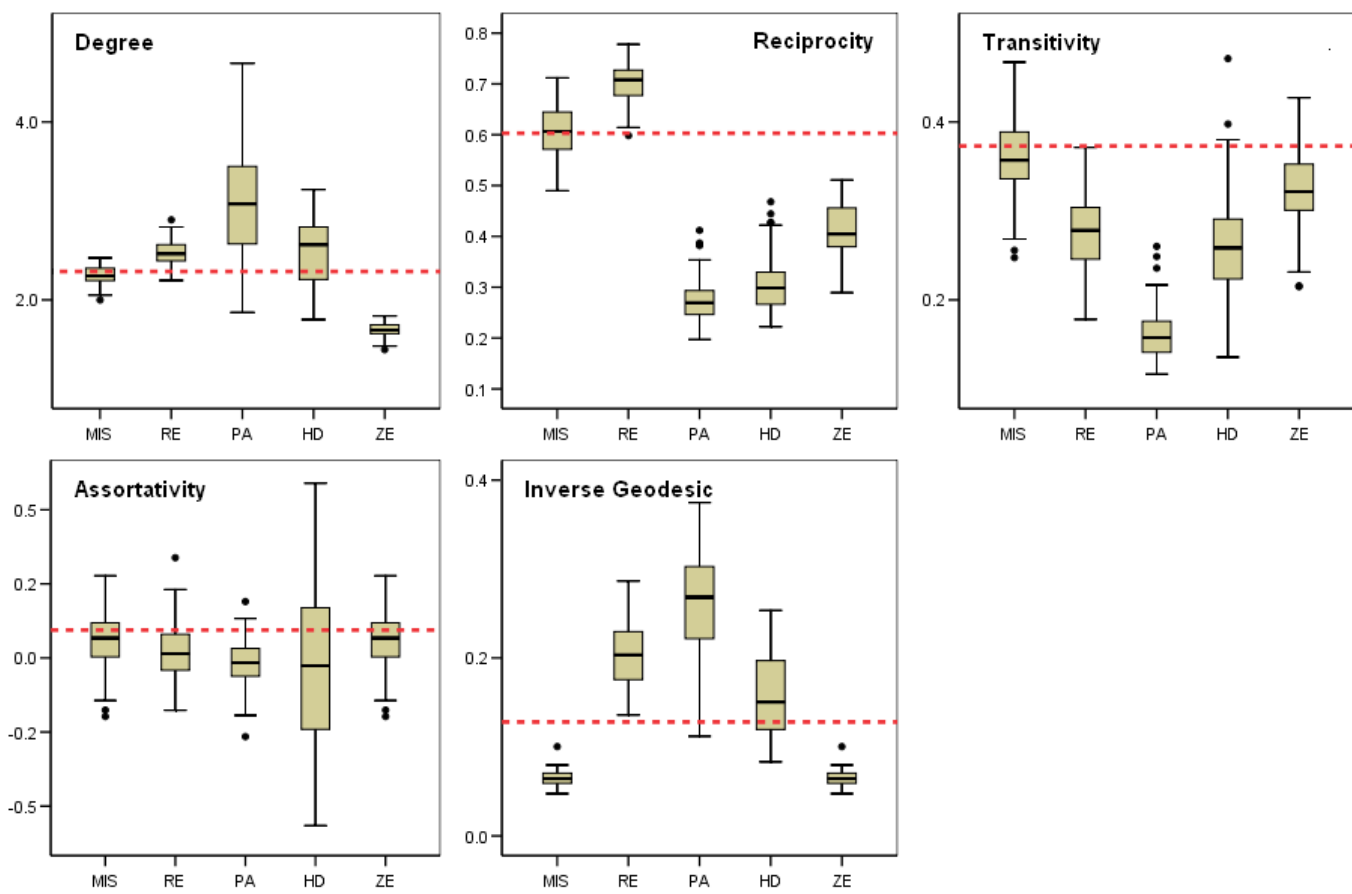

Figure 7: Box plots of the effects of missing data and imputation on the five network statistics mean degree, reciprocity, transitivity, assortativity, and mean geodesic distance in the example data. Within each plot the methods are: ignoring the missingness (MIS), reconstruction $(\mathrm{RE})$, preferential attachment $(\mathrm{PA})$, hot deck imputation (HD), and imputing zeros (ZE).

The effects shown in Table 4 are in line with the results of the simulation study (cf. the effects in figures 3 to 6 , for $20 \%$ unit non-response). The mean degree is larger for the reconstruction and hot deck imputations, and is much smaller for imputing zeros. Reciprocity is largest for reconstruction, and small for the other imputation methods. Transitivity is smaller for reconstruction and preferential attachment, but larger for hot deck imputation (where the simulations in Section 4 show smaller values). Assortativity is small for all methods, except reconstruction, and inverse geodesic distance is large.

Table 4: Four network statistics, mean degree (D), reciprocity $(\mathrm{R})$, transitivity $(\mathrm{T})$, assortativity $(\mathrm{A})$, and mean inverse geodesic distance $(\mathrm{G})$ computed for the incomplete and imputed example data.

\begin{tabular}{lcccrc}
\hline & $\mathrm{D}$ & $\mathrm{R}$ & $\mathrm{T}$ & \multicolumn{1}{c}{$\mathrm{A}$} & $\mathrm{G}$ \\
\hline Incomplete & 6.720 & 0.705 & 0.463 & 0.015 & 0.397 \\
Reconstruction & 7.156 & 0.786 & 0.443 & 0.033 & 0.540 \\
Pref. attachment & 6.469 & 0.483 & 0.396 & 0.004 & 0.529 \\
Hot deck & 9.094 & 0.545 & 0.471 & -0.056 & 0.541 \\
Imputing zeros & 5.250 & 0.512 & 0.460 & 0.015 & 0.397 \\
\hline
\end{tabular}

